\title{
ATEFI - Um algoritmo para controle semafórico em tempo fixo descentralizado
}

\section{ATEFI - A descentralized fix time algorithm for signal control}

\author{
Silvia Galvão de Souza Cervantes ${ }^{1}$; Juliani Chico Piai²; \\ Ernesto Fernando Ferreyra Ramírez; ${ }^{3}$ Lucas Varasquim'; Edmar Nagayama ${ }^{5}$
}

\section{Resumo}

Este trabalho tem como proposta a apresentação de um algoritmo descentralizado em tempo fixo (ATEFI), com a finalidade de fornecer planos de tempos semafóricos otimizados. Os resultados do algoritmo serão comparados com o software comercial TRANSYT. O objetivo é a minimização do atraso e a comparação de estratégias que buscam a solução para os problemas de gerenciamento de tráfego urbano a baixo custo e adaptados à realidade da malha viária nacional.

Palavras-chave: Engenharia de tráfego, Otimização, Controle semafórico.

\begin{abstract}
This work proposes the presentation of a decentralized fixed time algorithm (ATEFI), with the purpose of finding optimized traffic light times. The results of the algorithm will be compared with commercial software called TRANSYT. The objective is to minimize the delay and the comparison of different strategies, trying to obtain a low cost solution for the urban traffic management problems.
\end{abstract}

Key words: Traffic engineering, Optimization, Signal control.

1 Docente do Departamento de Engenharia Elétrica da Universidade Estadual de Londrina; silvia@uel.br Aluna de mestrado do Depto de Engenharia Elétrica, Universidade Estadual de Londrina; ju_piai@hotmail.com Docente do Departamento de Engenharia Elétrica da Universidade Estadual de Londrina; ferreyra@uel.br

4 Aluno de IC do Depto de Engenharia Elétrica, Universidade Estadual de Londrina; lucasvarasquim@uol.com.br

5 Aluno de IC do Depto de Engenharia Elétrica, Universidade Estadual de Londrina; ednagayama@gmail.com 


\section{Introdução}

Sistemas de gerenciamento de tráfego compostos por monitoramento da demanda veicular e estratégias de controle semafórico em tempo fixo e real fazem parte de um conjunto de soluções para os freqüentes problemas de congestionamentos que ocorrem hoje nas malhas viárias de cidades de grande e médio porte.

O controle de tráfego nas interseções semaforizadas busca proporcionar maior segurança à travessia de pedestres e veículos, através da melhora das condições de fluxo veicular com o objetivo de reduzir os desperdícios de tempo, combustível e minimizar o esforço empreendido nos deslocamentos diários dos habitantes e mercadorias (PAIVA NETO; CASTRO NETO; LOUREIRO, 2003).

Algumas capitais brasileiras, como São Paulo, Rio de Janeiro e Fortaleza, têm implantado sistemas centralizados de controle de tráfego urbano (CTA - Controle de Tráfego em Área) para promover gestão mais eficiente e eficaz dos deslocamentos viários (LOUREIRO; GOMES; LEANDRO, 2002). Estes sistemas são chamados Urban Traffic Control (UTC) que centralizam o monitoramento e o controle dos sinais de tráfego em suas jurisdições (HUNT; ROBERTSON; BRETHERTON, 1982), (ROBERTSON, 1969), (PECK; GORTON; LIREN, 1990).

No entanto, são poucas as cidades brasileiras que têm hoje infraestrutura para a implantação e gerenciamento de centrais de controle de tráfego urbano automatizadas. Este trabalho apresenta algumas etapas de uma pesquisa que está sendo desenvolvida na Universidade Estadual de Londrina, com o objetivo de criar um sistema de gerenciamento de tráfego, que possa acoplar algoritmos em tempo real e tempo fixo atendendo subsistemas da malha viária de forma coordenada.

Os algoritmos de controle que fazem parte das estratégias de gerenciamento podem ser classificados em três grupos: tempo fixo, seleção dinâmica e tempo real (AKCELIK, 1988). Elas se diferenciam basicamente quanto ao grau de sensibilidade às variações da demanda. O controle de tempo fixo se caracteriza pela utilização de planos précalculados, com base no volume médio de tráfego, em determinados períodos do dia. A estratégia de seleção dinâmica identifica, dentre um conjunto de planos de tempo fixo pré-definidos, aquele que melhor se ajusta às condições detectadas. Já no controle em tempo real, a programação semafórica é calculada dinamicamente, segundo demanda de tráfego detectada em tempo real (PAIVA NETO; CASTRO NETO; LOUREIRO, 2003).

A proposta deste artigo é apresentar um algoritmo em tempo fixo (ATEFI - Algoritmo em Tempo Fixo), baseado na estratégia TRANSYT/10 (CRABTREE; VINCENT; HARRISON, 1996). A primeira etapa com o objetivo de validar o algoritmo é a comparação de resultados com o TRANSYT. Este trabalho está inserido em um projeto maior cujo objetivo é o desenvolvimento de uma estratégia de gerenciamento de tráfego de baixo custo de implantação, manutenção e adequada à realidade nacional.

\section{Sistema TRANSYT}

O TRANSYT (TRAFFIC NETWORK StUdY TOOL) é um software que utiliza uma estratégia de otimização offline para calcular tempos semafóricos otimizados em uma rede de tráfego. Desde a primeira estratégia, desenvolvida em 1967 no Regional Research Laboratory (RRL), Inglaterra (ROBERTSON, 1969), várias versões têm sido produzidas. A mais recente delas é o TRANSYT 13 (BINNING; BURTENSHAW, 2008).

A estratégia TRANSYT é composta de um modelo de tráfego e um modelo de otimização, Figura 1. A função objetiva do modelo de otimização considera o peso combinado do número de paradas e o atraso sofrido pelos veículos em todas as aproximações da rede semaforizada. Conceitualmente, o TRANSYT gera programações semafóricas que beneficiam o 
conjunto de toda a subárea, mas não necessariamente fornece progressão satisfatória para as aproximações da via arterial (PAIVA NETO; CASTRO NETO; LOUREIRO, 2003).

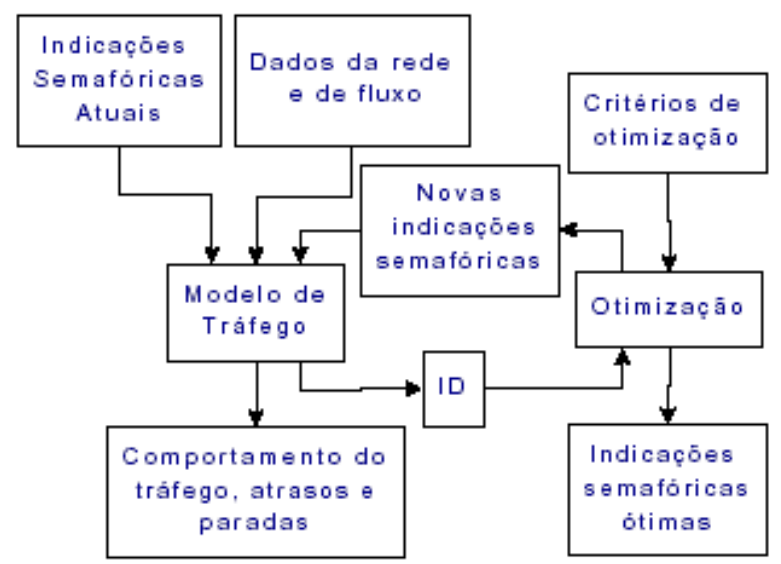

Figura 1. Modelo do TRANSYT.

A Figura 1 apresenta o bloco de dados de entrada que são características da rede, como: demanda fixa de veículos, número de interseções, número de vias, etc; outras entradas são as indicações semafóricas iniciais. Com estes dados o modelo de tráfego, calcula o índice de desempenho (ID) para a rede (RAKHA; VAN AERDE, 1996). Através do ID avalia-se o congestionamento do tráfego (ROBERTSON, 1969), considerando-se os atrasos e paradas, obtendo-se os histogramas para cada ciclo da rede.

O processo de otimização é baseado na heurística Hill Climbing, pois os dados de demanda veicular são conhecidos antecipadamente. Os dados necessários para otimização, ou seja, as restrições do sistema, são os valores mínimos dos tempos de verde, ciclo e os custos. Os tempos semafóricos são ajustados inicialmente e, aplicados ao modelo de tráfego, calcula-se o tempo de ajuste que promove o melhor ID. Por adoções sucessivas de tempos benéficos, o ótimo é encontrado. Este valor ótimo é aplicado ao modelo de tráfego que apresenta o comportamento veicular otimizado nas vias (ROBERTSON, 1969).

\section{Metodologia}

O enfoque das pesquisas em controle de tráfego hoje se concentra em algoritmos de controle em tempo real. No entanto, muitas cidades de médio e pequeno porte sofrem com os problemas de falta de gerenciamento de suas malhas viárias e não conseguem justificar o investimento em sistemas em tempo real. Este algoritmo em tempo fixo visa melhorar as condições de tráfego nestas cidades de forma eficiente e econômica.

O ATEFI é caracterizado como determinístico e descentralizado. Determinístico porque inseridos os dados de entrada, existe um único caminho de processamento e um único resultado final e, descentralizado, pois, o cálculo dos tempos semafóricos é realizado para uma interseção por vez, independente das interseções vizinhas. Sistema similar foi desenvolvido em (VILANOVA, 2006).

As áreas avaliadas têm um tempo de ciclo comum. O ciclo é dividido em um número de intervalos iguais, usualmente de um a três segundos de duração. Os cálculos são realizados com base na média de taxa de fluxo representados em histogramas.

É composto por um modelo de tráfego e um modelo de otimização. O modelo de tráfego simula as condições da rede, para isso, algumas suposições são feitas respeitando condições impostas pelo usuário como o tempo de ciclo e tempo de verde mínimo. Equações de estado definem a formação da fila, o atraso e o controle dos semáforos formadores da rede de tráfego. O modelo de otimização calcula o índice de desempenho da rede e, através da heurística Hill Climbing (GOLDBERG, 1989), determina o tempo de verde e a defasagem ótima, reduzindo assim o atraso veicular.

Planos semafóricos são criados para diferentes horários do dia e dias da semana. Desta forma, garante-se a adequação dos tempos semafóricos às alterações da demanda veicular na rede.

O algoritmo encontra-se em constante evolução: estão sendo testadas novas estratégias de otimização, 
sua generalização para qualquer configuração de malha viária. Também a otimização do tempo de ciclo, bem como o entreverdes, já se encontram em fase de desenvolvimento.

Um projeto em fase de avaliação busca recursos para compra de um microsimulador para validação do algoritmo.

\section{Modelo de Tráfego}

A rede é formada por duas entidades: os nós, que representam as interseções semaforizadas, e as vias, que representam os fluxos unidirecionais de veículos entre dois nós. A via pode ser do tipo entrada ou interna. A primeira é aquela que provém de um local externo à rede e desemboca em um de seus nós. A via interna parte de um nó denominado nó origem e chega a outro, denominado nó destino. Os veículos percorrem as vias em uma velocidade informada e se detêm quando encontram o nó destino em uma situação de indicação semafórica vermelha, ou então quando alcançam uma fila de veículos parados.

Nas vias de entrada, adotou-se um perfil de chegada fixo, distribuído uniformemente no tempo. Esta escolha deve-se a necessidade de reprodutividade dos resultados para comparações. Já nas vias internas existem duas seções: uma a montante e outra a jusante. $\mathrm{O}$ algoritmo calcula as chegadas na seção à jusante a partir da seção a montante como descrito na Equação 1,

$$
z_{l}(t)=\sum_{\substack{l^{\prime} \in \boldsymbol{U} l \\ p_{l}, l}} \min \left(m_{l^{\prime}}(t)+z_{l^{\prime}}(t), \text { s.c. } c_{l^{\prime}}(t)\right)
$$

onde $z_{l}(t)$ representa a chegada de veículos em um intervalo $t ; l$ ' $\mathrm{E} U_{l}$ é o conjunto de vias a montante de $l ; p_{l^{\prime} l}$ a proporção de veículos que convergem de $l$ ' para $l ; m_{l},(t)$ representa o tamanho da fila no intervalo $t ; s$ a taxa de descarga da via, constante $\mathrm{e}$ igual a dois por faixa e $c_{l},(t)$ a variável de controle na via $l$ ' $\operatorname{com} c_{l}(t) \mathrm{E}\{0,1\}$.
Para impedir os conflitos entre vias, a indicação semafórica deve ser complementar, visto que o sistema em questão é composto por duas fases: verde (0) e vermelho (1). Tal relação é definida por equações de estado:

$$
c_{l}(t)=v_{i}(t) \text { vias arteriais }
$$

$$
c_{l}(t)=v_{i}(t) \text { vias secundárias }
$$

onde $v_{i}(t)$ representa a indicação semafórica vigente na interseção $i$.

O ATEFI incrementa a fila dos veículos em cada via à medida que eles vão se acumulando. Duas situações de incremento podem ocorrer: durante o tempo de vermelho ou devido à existência de veículos parados antes da linha de retenção, Equação 3.

$$
m_{l}(t+1)=\max \left[0, m_{l}(t)+z_{l}(t+1)-s . c_{l}(t+1)\right]
$$

A informação contínua da situação das filas permite calcular o ID do modelo que é determinado, neste caso, pelo atraso. Estudos comprovam que o peso do número de paradas no cálculo do ID é muito menor que o do atraso. Situações podem ocorrer onde o critério de número de paradas privilegia uma quantidade menor de veículos já parados a uma quantidade maior que se aproximam da linha de retenção. Este cenário é um exemplo da dificuldade na determinação de pesos na combinação de diferentes critérios. Dessa forma, justifica-se desprezar o parâmetro de número de paradas sem perdas significativas no resultado. Um estudo mais detalhado pode ser encontrado em (CERVANTES, 2005). O desempenho da rede é calculado pela Equação 4. 


$$
d(t)=\sum_{\mathrm{j}=t+1}^{\mathrm{T}} m_{l}(j)
$$

onde $\mathrm{d}(\mathrm{t})$ é o atraso total e T o tempo de simulação.

O modelo de tráfego apresentado considera condições não saturadas, o que caracteriza um número máximo de 1800 veículos por faixa na interseção em uma hora.

\section{Otimização}

O problema de otimização consiste em dado o modelo que representa os estados do tráfego urbano, encontrar a seqüência de valores de controle que minimize o atraso veicular.

Dentre os valores a serem considerados nesta etapa estão o tempo de ciclo, considerado igual e constante para todos os nós da rede, o conjunto das distribuições para cada nó desse tempo de ciclo em tempos de verde/vermelho e o índice de desempenho da rede. A distribuição entre fases verde/vermelho respeita o limite mínimo do tempo de verde para cada via. Esta restrição é testada a cada iteração em um laço de comparação.

A heurística de otimização aplicada na busca do tempo de verde que garanta o menor atraso é a Hill Climbing. É uma técnica de otimização que pertence à família de busca local. Embora técnicas mais avançadas possam oferecer melhores resultados, o Hill Climbing é simples de implementar e funciona bem em diversas situações. A técnica busca sempre maximizar (ou minimizar) uma função $f(x)$, onde $x$ são estados discretos. Estes são tipicamente representados por vértices em um gráfico. A variável $x$ tem um estado inicial aleatório, e é alterada de modo que se obtenha um aumento (ou diminuição) no valor de $f(x)$, até que um máximo (ou mínimo) local seja encontrado. Quando se deseja trabalhar em um espaço contínuo, o algoritmo leva o nome de Gradient Ascent (para maximização) ou Gradient Descent (minimização). Esta técnica pode ser aplicada a problemas que apresentem várias soluções. Neste caso, não é possível garantir que a solução encontrada é um ótimo global. Outra desvantagem é que no universo de busca pode ocorrer é uma planície (região plana da função), em que o algoritmo não consegue definir em que direção deve incrementar as variáveis, pois nenhuma decisão causará maximização (ou minimização) da solução (MICHALEWICZ; FOGEL, 2000).

Para a otimização do tempo de verde inicialmente considera-se uma configuração de tempos semafóricos proposta. São realizados iterações com incrementos nos tempos até a obtenção de uma solução ótima. O ID da rede é inicialmente calculado para um ajuste de tempo semafórico igual ao tempo de verde mínimo. Posteriormente, são implementados incrementos ao tempo de verde (decrementos para a via em conflito) e um novo ID é calculado. É realizada uma comparação entre o ID obtido na iteração atual e o menor ID até o momento. Este tempo semafórico é fixado para a interseção em estudo e torna-se condição inicial para o cálculo das chegadas veiculares nas vias internas. O processo termina quando todos os nós foram verificados e as indicações ótimas encontradas.

Um pseudocódigo do algoritmo que descreve o processo de otimização Hill Climbing aplicado ao controle de tráfego é apresentado a seguir.

$I D=$ infinito;

PARA (ind_semaf DE v_min ATÉv_max)

Calcula_fila (ind_semaf);

ID_atual = calcula_ID (ind_semaf);

$S E$ (ID_atual $\angle I D) I D=I D \_a t u a l$;

SENÃO sai do laço;

Onde ind_semaf representa a indicação semafórica atual; $v \_$min o tempo de verde mínimo; $v \_$max o tempo de verde máximo; calcula_fila representa o cálculo da fila e $I D$ o índice de desempenho. 
Na otimização do tempo de defasagem é calculado o ID da rede para uma configuração inicial dos tempos semafóricos. O programa altera então a defasagem de um dos nós em um incremento pré-determinado, e recalcula o ID da rede. Se o indicador diminui, a defasagem é alterada sucessivamente na mesma direção até que um ID mínimo seja encontrado. Se a mudança inicial causar um aumento no ID, a defasagem é alterada então na direção oposta, até que um valor mínimo do ID seja encontrado. $\mathrm{Na}$ estratégia usada pelo TRANSYT (com o objetivo de sair de uma região de planície) é montada uma lista com diferentes tamanhos para o incremento. $\mathrm{O}$ primeiro incremento da lista é utilizado no cálculo do ID para cada nó, seqüencialmente. Depois de otimizado o último nó, o processo é reiniciado, até que todos os valores da lista sejam aplicados. Esse procedimento resulta em diferentes soluções para o problema, e a melhor solução é armazenada. Com incrementos diferentes, é possível escapar da planície, desde que algum valor da lista seja maior do que o tamanho dessa planície (CRABTREE; VINCENT; HARRISON, 1996).

No ATEFI os incrementos utilizados são de tamanho fixo, e para contornar os problemas apresentados é utilizada uma técnica chamada de Shotgun Hill Climbing, ou Random-Restart Hill Climbing. Esta técnica trabalha com iterações em cima do algoritmo Hill Climbing, mudando aleatoriamente as condições iniciais do problema a ser resolvido. Deste modo torna-se possível escapar das planícies e obter pontos que levem a diferentes máximos (ou mínimos) dentro da função. Comparam-se então as soluções, e escolhe-se dentre elas qual a melhor.

\section{Estudo de Caso}

Através de contagens manuais, a Prefeitura de Londrina/PR, representada pelo Instituto de
Pesquisa e Planejamento Urbano de Londrina (IPPUL), obteve o fluxo veicular da região central. Estas contagens foram fornecidas ao Departamento de Engenharia Elétrica da Universidade Estadual de Londrina através de convênio firmado visando pesquisa para a melhoria das condições de tráfego da cidade.

Com o objetivo de testar o algoritmo, foram feitas simulações considerando a Rua Senador Souza Naves (SN) e suas interseções com as Ruas Espírito Santo (ES), Goiás (GO) e Pará (PA) como apresentado na Figura 2. Na Figura 3 apresenta-se um mapa da região estudada. Todas as vias são de mão única com duas faixas para tráfego e uma para estacionamento. Os testes foram realizados para uma rede parcial de pequeno porte para facilitar a reprodução de resultados e aperfeiçoamento do algoritmo. Novas simulações estão sendo realizadas para uma rede de 6 interseções, apresentando ciclos entre elas.

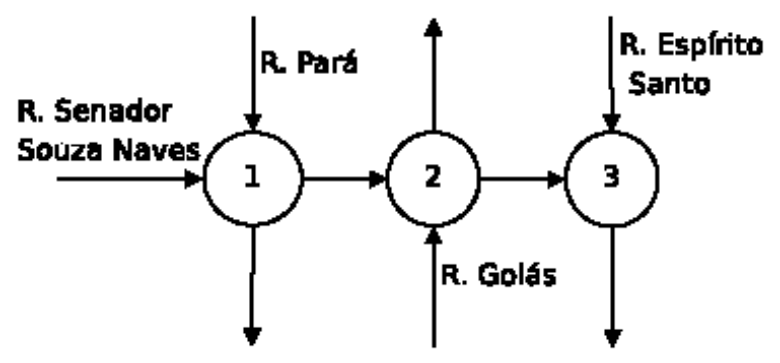

Figura 2. Rede de Tráfego.

Contabilizando uma média de veículos por hora chegando nas vias com base nos dados fornecidos pelo IPPUL, é possível obter a entrada veicular das vias de entrada. Serão considerados dois períodos do dia nas simulações: um das dezessete horas e trinta minutos às dezoito horas e trinta minutos, de alto carregamento, e outro das nove horas e trinta minutos às dez horas e trinta minutos, de médio carregamento, como mostra a Tabela 1. 


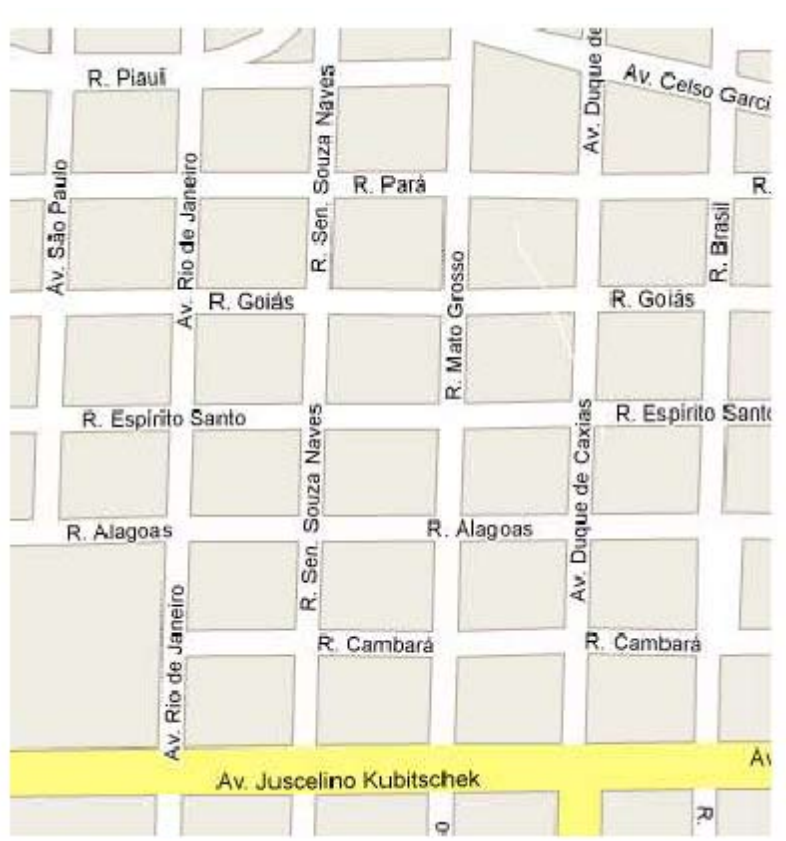

Figura 3. Mapa parcial da região central de Londrina Fonte: IPPUL.

Tabela 1. Fluxos de tráfego por períodos do dia (veic/h).

\begin{tabular}{ccccc}
\hline & SN & ES & GO & PA \\
\hline Alto & 1255 & 611 & 1384 & 1340 \\
Médio & 1049 & 411 & 1322 & 851 \\
\hline
\end{tabular}

De acordo com a Figura 2 pode-se notar que os veículos circulantes têm a possiblidade de converter à direita $(\mathrm{CD})$ ou à esquerda $(\mathrm{CE})$ nos nós, de acordo com o sentido das vias. Dessa forma, a Tabela 2 apresenta a proporção de veículos nas vias que convergem de acordo com o sentido permitido no local.

Tabela 2. Proporção de conversão entre vias.

\begin{tabular}{ccc}
\hline & \multicolumn{2}{c}{ SN } \\
\hline & CD & CE \\
\hline ES & $22 \%$ & $28 \%$ \\
GO & $27 \%$ & $30 \%$ \\
PA & $28 \%$ & $27 \%$ \\
\hline
\end{tabular}

O tempo de verde mínimo estabelecido, seguindo a mesma proporção adotada pelo TRANSYT, é 12 segundos. O tempo total de simulação será de uma hora, subdividida em passos de 3 segundos (tempo amostral). Como o tamanho do ciclo influencia diretamente no valor do atraso, a implementação desta otimização está em fase de desenvolvimento e fará parte da versão final do algoritmo. No entanto, simulações para diferentes tamanhos de ciclo foram realizadas e seus resultados serão apresentados a seguir.

\section{Resultados}

Os índices de desempenho, tanto para condições de alto carregamento, Tabela 3, quanto para médio, Tabela 4, obtidos na simulação do ATEFI serão comparados ao do TRANSYT/10 (licença acadêmica). Sabendo-se da influência do tempo de ciclo na obtenção do melhor desempenho da rede, simulações para ciclos de 45, 60 e 90 segundos são apresentadas para alto e médio carregamento do tráfego.

Tabela 3. Atraso (veic.-h/h) ATEFI x TRANSYT - Alto Carregamento.

\begin{tabular}{ccccc}
\hline Ciclo (s) & & SNxPA & SNxGO & SNxES \\
\hline \multirow{2}{*}{45} & ATEFI & 6,3 & 5,2 & 2,7 \\
& TRANSYT & 8,1 & 7,4 & 3,4 \\
\hline \multirow{2}{*}{60} & ATEFI & 8,4 & 7,5 & 3,8 \\
& TRANSYT & 10,2 & 9,2 & 4,2 \\
\hline \multirow{2}{*}{90} & ATEFI & 12,6 & 11,4 & 5,6 \\
& TRANSYT & 14,5 & 12,7 & 6,0 \\
\hline
\end{tabular}

Tabela 4. Atraso (veic.-h/h) ATEFI x TRANSYT - Médio Carregamento.

\begin{tabular}{ccccc}
\hline Ciclo (s) & & SNxPA & SNxGO & SNxES \\
\hline \multirow{2}{*}{45} & ATEFI & 4,0 & 4,1 & 1,8 \\
& TRANSYT & 4,7 & 5,6 & 2,3 \\
\hline \multirow{2}{*}{60} & ATEFI & 5,3 & 6,0 & 2,3 \\
& TRANSYT & 6,1 & 6,9 & 2,7 \\
\hline \multirow{2}{*}{90} & ATEFI & 7,9 & 9,2 & 3,5 \\
& TRANSYT & 8,8 & 9,5 & 3,6 \\
\hline
\end{tabular}


Foi considerado um perfil de chegadas fixo uniformemente distribuídos. Assim, pela característica de existência de um ciclo fixo no ATEFI, os veículos têm um período para acomodação e formação de pelotões. Estes podem resultar em um perfil próximo ao de uma demanda pulsada. No TRANSYT sabe-se que a chegada é considerada aleatória, no entanto, não se pode definir o mesmo perfil de entrada para comparação dos algoritmos.

Os resultados das Tabelas 3 e 4 mostram que para o caso em estudo, o ciclo que apresenta o menos valor de atraso é o de 45 segundos. Esperava-se que para alto carregamento um tamanho de ciclo maior apresenta-se maiores resultados, já que valores maiores de fluxo necessitam de ciclos maiores para aumentar a capacidade e evitar o congestionamento. No entanto, não se percebe este comportamento no algoritmo pelos resultados apresentados. Após a implementação da rotina de otimização de tamanho de ciclo novas simulações serão efetuadas para comparação dos resultados. Também estudos mais aprofundados quanto aos resultados da simulação serão realizados. Para médio carregamento, onde os valores de fluxo são menores, esperava-se um ciclo menor. Este comportamento pode ser comprovado na Tabela 4.

Os resultados do ATEFI e o TRANSYT são próximos. Este resultado era esperado, pois as estratégias são similares e buscam o mesmo desempenho.

\section{Conclusões}

A proximidade dos resultados do ATEFI com a estratégia TRANSYT, indica que o modelo de tráfego e o modelo de otimização desenvolvidos neste trabalho são os primeiros passos na direção de uma ferramenta para obtenção de planos a tempo fixo para controle de tráfego de baixo custo e adequada à realidade nacional.

Assim, considera-se a avaliação do algoritmo, até esta fase, satisfatória. Novos testes com outras configurações de sistema serão realizados, bem como a generalização da rede, do número de laços e estágios, além da busca de uma otimização que garanta a obtenção de um ótimo global.

\section{Agradecimentos}

Os autores agradecem a CAPES pelo financiamento da bolsa de mestrado da co-autora. À Fundação Araucária e UEL pelas bolsas dos alunos de IC, à Prefeitura de Londrina através de seu órgão IPPUL, pelo fornecimento dos dados necessários para realização das simulações e à UEL por proporcionar a realização deste trabalho.

\section{Referências}

AKCELIK, R. The Highway Capacity Manual delay formula for signalized intersections. ITE Journal, v. 58, n. 3, p. 23-27, 1988.

BINNING, J.; BURTENSHAW, G. Green light for TRANSYT 13. Traffic Software News, UK, http://www. trlsoftware.co.uk/files/general/TRANSYT13 Features. pdf, acessado em 16 de abril de 2008.

CERVANTES, S. G. S. Um Algoritmo Descentralizado para Controle de Tráfego Urbano em Tempo Real. Tese (Doutorado) - Universidade Federal de Santa Catarina, Florianópolis, Santa Catarina, Agosto 2005.

CRABTREE, M. R.; VINCENT, R. A.; HARRISON, S. TRANSYT/10 User Guide. Application guide 28. Crowthorne, Berkshire, 1996.

GOLDBERG, D. E. Genetic algorithms in search optimization and machine learning. [S.1.]: AddisonWesley, 1989.

HUNT, P. B.; ROBERTSON, D. L.; BRETHERTON, R. D. The SCOOT on-line traffic signal optimization technique. Traffic Eng. Control, v. 23, p. 190-192, 1982.

LOUREIRO, C. F. G.; GOMES, M. J. T. L.; LEANDRO, C. H. P. Avaliação do desempenho nos períodos de pico do tráfego de interseções semaforizadas com controle centralizado em tempo fixo e real. In: XVIANPET - CONGRESSO DE PESQUISA E ENSINO EMTRANSPORTES. [S.1.]: Revista de Pesquisa e Ensino em Transportes, 2002. v. 1, p. 365-376.

MICHALEWICZ, Z.; FOGEL, D. B. How To Solve It: Modern Heuristics. Berlin: Springer-Verlag, 2000. 
PAIVA NETO, P. M.; CASTRO NETO, M. M.; LOUREIRO, C. F. G. Progressão em tempo real versus tempo fixo por banda de passagem em períodos de baixa demanda - estudo de caso. In Proc.: ANPET: XVII Congresso de Pesquisa e Ensino em Tranportes, Rio de Janeiro-RJ, p. 1-8, 2003.

PECK, C.; GORTON, P. T. W.; LIREN, D. The application of scoot in developing countries. In: THIRD INTERNATIONAL CONFERENCE ON ROAD TRAFFIC CONTROL - IEE. London, UK, 1990.
RAKHA, H. A.; VAN AERDE, M. W. A comparison of the simulation modules of the TRANSYT and INTEGRATION models. Transportation Research Record, v. 1566, p. 1-7, 1996.

ROBERTSON, D. I. TRANSYT: A Traffic Network Study Tool. [S.l.], maio 1969.

VILANOVA, L. M. Siri - um novo simulador para redes de semáforos. ITS Panamericano, 2006. 
